\title{
The association of continuity of care and cancer centre affiliation with outcomes among patients with cancer who require emergency department care
}

\author{
Keerat Grewal MD MSc, Rinku Sutradhar PhD, Monika K. Krzyzanowska MD MPH, \\ Donald A. Redelmeier MD MSc, Clare L. Atzema MD MSc
}

Cite as: CMAJ 2019 April 23;191:E436-45. doi: 10.1503/cmaj.180962

Visual abstract available at www.cmaj.ca/lookup/suppl/doi:10.1503/cmaj.180962/-/DC2

\begin{abstract}
BACKGROUND: Patients with cancer have complex care requirements and frequently use the emergency department. The purpose of this study was to determine whether continuity of care, cancer expertise of an institution or both affect outcomes in patients with cancer in the emergency setting.
\end{abstract}

METHODS: We conducted a retrospective cohort study using administrative databases from Ontario, Canada, involving records of patients aged 20 years and older who received chemotherapy or radiation in the 30 days before a cancerrelated visit to the emergency department between 2006 and 2011. Patients seen in an emergency department at an alternative hospital (not the site where cancer treatment was given) were matched based on propensity score to patients who visited their original hospital (site where cancer treatment was given). Next, patients seen at an alternative emergency department that was in a general hospital (i.e., not a cancer centre) were matched to patients who visited their original hospital or a cancer centre. Outcomes were admission to hospital at the index visit to the emergency department, 30-day mortality, having imaging with computed tomography and return visits to the emergency department.

RESULTS: We found 42820 patients who were eligible for our study. Patients seen in the emergency departments at alternative hospitals were less likely to be admitted to hospital (odds ratio [OR] 0.78, 95\% confidence interval $[\mathrm{Cl}] 0.74-0.83$ ) and had higher hazards of return visits to the emergency department than matched patients at original hospitals (hazard ratio [HR] 1.06, 95\% Cl 1.03-1.11). In comparison, patients at alternative general hospitals also had lower odds of admission to hospital (OR 0.83, 95\% Cl 0.79-0.88) and higher hazards of return visits to the emergency department (HR 1.07, 95\% Cl 1.03-1.11) compared with matched counterparts; however, these patients had higher 30-day mortality (OR 1.13, 95\% Cl 1.05-1.22) and lower odds of having CT imaging (OR 0.74, 95\% Cl 0.69-0.80).

INTERPRETATION: Cancer expertise of an institution rather than continuity of care may be an important predictor of outcomes following emergency treatment of patients with cancer. ancer is one of the leading causes of mortality worldwide, and patients with cancer frequently use the emergency department. ${ }^{1-5}$ Previous studies have shown the importance of continuity of medical care to patient outcomes in certain patient populations. ${ }^{6-10}$ This may be particularly relevant for patients who are undergoing active treatment for cancer, because these patients have complex care requirements. ${ }^{11,12}$ Poor continuity of care, as a result of a visit to an emergency department at an alternative hospital (i.e., not the hospital where cancer treatment is received), could be associated with worse outcomes secondary to lack of familiarity with the patient's course of illness. Information gaps owing to poor continuity of care may lead to treatment delays or receipt of duplicate or unnecessary investigations and treatment.

Lack of subspecialized expertise could also be a factor in emergency department care: cancer expertise has been associated with better outcomes among patients who receive surgical and medical cancer care. ${ }^{11,13-16}$ Emergency departments without access to subspecialized cancer expertise may not be able to offer the same level of care.

We aimed to determine whether continuity of care, cancer care expertise of the institution or both are associated with outcomes in patients with cancer in the emergency department setting. 


\section{Methods}

We conducted a retrospective analysis of population-based administrative data from Ontario, Canada, between Apr. 1, 2006, and Mar. 31, 2011.

\section{Data sources}

We used Ontario health administrative databases held at ICES to identify study patients and obtain related information (Appendix 1, available at www.cmaj.ca/lookup/suppl/ doi:10.1503/cmaj.180962/-/DC1, provides a description of these databases). Patients were linked to the databases using a unique encoded identifier. Ontario has universal health care coverage for medically necessary care, therefore these databases contain most instances of health care utilization in the province.

\section{Study participants}

We identified adult patients with cancer aged 20 years and older from the Ontario Cancer Registry if they had a valid Ontario Health Insurance Plan health card number. To be included in the study, patients must have received chemotherapy or radiation in the outpatient setting in the 30 days before a visit to the emergency department. We examined data for only the first (index) cancer-related visit to the emergency department found in the National Ambulatory Care Reporting System (Canadian Institute for Health Information) during the study period.

To identify cancer-related visits to the emergency department, we used a previously validated approach to identify chemotherapy-related codes using International Statistical Classification of Diseases and Related Health Problems, 10th Revision (ICD-10) codes. ${ }^{17}$ However, the validation study was performed only in patients with breast cancer; therefore, we expanded our approach to be more generalizable and included all cancers, and both chemotherapy and radiation therapy. Specifically, we added ICD-10 codes to capture adverse effects and toxicities related to radiation therapy, based on common adverse events reported in the Common Toxicity Criteria Manual. ${ }^{18}$ Furthermore, we also examined the patient's "chief complaint" or reason for the visit to the emergency department (Supplemental Table 1, Appendix 1). The datapoint chief complaint in the National Ambulatory Care Reporting System was not mandatory as of fiscal year 2011; therefore, we cut off the study timeframe at this year.

We excluded patients seen in an Ontario emergency department that was not open 24 hours per day. We also excluded patients who died in the emergency department or who left the emergency department without being seen, because they were not available for the primary outcome measure (hospital admission).

\section{Exposure variables of interest}

Our first exposure variable of interest was the type of emergency department visit: a visit to the emergency department of an original or an alternative hospital. We defined a visit to an original hospital as a visit to an emergency department associated with the institution where the patient received chemotherapy or radiation therapy. We defined a visit to an alternative hospital as a visit to an emergency department that was not associated with the institution where the patient received chemotherapy or radiation therapy (thus, an alternative hospital could include other cancer centres).

To differentiate the effect of care continuity from cancer expertise, we examined a second exposure variable of interest: whether patients were seen at an alternative emergency department that was in a general hospital (i.e., not a cancer centre) versus patients who were seen at an original hospital or another cancer centre. A cancer centre was defined as 1 of the 14 major cancer centres in Ontario.

Each regional cancer centre in Ontario provides care that complies with provincial standards. ${ }^{19}$ Furthermore, regional cancer centres provide oversight and coordinate cancer care with partner hospitals in their region. Although there are 14 regional cancer centres, there are over 70 facilities in Ontario that provide chemotherapy, a substantial proportion of which do so under the guidance of regional cancer centres, and they are considered satellite cancer centres.

We considered patients who received chemotherapy and radiation simultaneously but at different institutions to have visited an original hospital if the visit to the emergency department occurred at either institution. We treated multi-institutional hospitals as a single facility because hospitals within 1 facility often share personnel, information and computer systems. Because we were specifically interested in the effect of emergency department care on outcome, we did not account for betweenhospital transfers for admitted patients in the analysis: all outcomes were attributed to the initial emergency department at which patients were seen.

\section{Outcomes}

Our primary outcome was admission to hospital at the index visit to the emergency department. Secondary outcomes were 30-day mortality, undergoing computed tomography (CT) and return visits to the emergency department within 30 days of discharge from the emergency department or hospital. Computed tomography was included as an outcome because we hypothesized that imaging practices may differ if a patient is known to an institution or if health care providers seeing the patients are familiar with typical complications of cancer.

\section{Covariates}

Model covariates included demographics (age, sex, income quintile and rural residence); noncancer comorbidities; previous health care use (admissions to hospital and visits to the emergency department in the previous 2 years), John Hopkins Aggregated Diagnosis Group (ADG) scores, which measure health care resource use in ambulatory populations to adjust for illness severity ${ }^{20}$ ); cancer-related variables (cancer type [solid or hematological], time since cancer diagnosis, type of treatment [chemotherapy, radiation or combination], palliative care); details of the visit to the emergency department (triage score [using the mandatory Canadian Triage and Acuity Scale, which measures illness acuity on presentation using a scale of 1 to 5], ${ }^{21}$ arrival by ambulance, time of visit, day of visit and cancer centre affiliation 
[for examining the first exposure variable]). For analyses examining the secondary outcomes, disposition of the emergency department was included as a covariate in the model. Appendix 1 provides further information about covariates.

\section{Statistical analysis}

We used descriptive statistics to characterize the type of emergency department visit. Univariate testing was performed on categorical and nonnormally distributed continuous variables using the $\chi^{2}$ test and the Wilcoxon rank sum test, respectively.

To ensure that patients were similar in their illness acuity on arrival at the emergency department, we used propensity scores to match patients with different types of visits to the emergency department. We used logistic regression models that included the covariates described above to estimate the propensity score, defined as the probability of visiting an alternative versus original emergency department and an alternative general emergency department versus an original emergency department or cancer centre. Patients were then matched on the logit of the propensity score, using 1:1 greedy matching, without replacement, and a caliper width of 0.2 of the standard deviation (SD) of the logit of the propensity score. ${ }^{22}$ We evaluated balance in baseline covariates by examining calculated standardized differences. ${ }^{23}$ The McNemar test was used to test differences in proportions for admission to hospital between matched groups.

We implemented a logistic regression incorporating the main exposure and accounting for the paired nature of the data to obtain odds ratios (ORs) and confidence intervals (Cls). Secondary outcomes (30-d mortality and imaging with $\mathrm{CT}$ ) were also assessed with the propensity score-matched cohort, but emergency department disposition was also included in the propensity score. To assess the effect of the match rate between propensity score-matched groups, we conducted a sensitivity analysis using inverse propensity-treatment weighting.

We examined the adjusted association of the independent variables of interest with return visits to the emergency department within 30 days using a subdistribution hazard model, which allowed us to account for the competing risk of death. For patients who were admitted to hospital at the index visit to the emergency department, only those who were alive when discharged from hospital were retained in the analysis of return visits to the emergency department.

Sensitivity analyses were conducted to examine all-cause visits and repeat visits to the emergency department.

All analyses were conducted using SAS 9.3.

\section{Ethics approval}

The study was approved by the Research Ethics Board at ICES.

\section{Results}

There were 42820 unique patients who were seen in the emergency department for a cancer-related reason within 30 days of receiving chemotherapy or radiation therapy (Figure 1). Of these, 16532 patients (38.6\%) were seen at an alternative emergency department (Table 1). A greater proportion of patients with a high acuity triage score (Canadian Triage and Acuity Scale level 1 or 2) were seen at original hospitals. Among patients seen at alternative hospitals, $1841(11.1 \%)$ were seen at a major cancer centre; therefore, in the cohort for examining the second independent variable of interest, 14691 (34.3\%) visited an alternative general hospital. Appendix 1 provides further description of the study population.

In the propensity score-matched analysis, 10273 patients (62.1\%) who visited an alternative emergency department were successfully matched to patients who visited an original emergency department (Table 2). Standardized differences were less than 0.10 for all covariates, indicating that the groups were well matched. For secondary outcomes, we rematched patients to include hospital admission at the index visit into the propensity score. Again, patients were well matched (Supplemental Table 2, Appendix 1). Patients seen at alternative emergency departments had significantly lower odds of admission to hospital (OR $0.78,95 \% \mathrm{Cl} 0.74-0.83$ ), relative to those seen at original emergency departments, with no difference in 30-day mortality or CT imaging (Table 3).

ED visits within $30 \mathrm{~d}$ of

chemotherapy or

radiation treatment in

Ontario from Apr. 1, 2006,

to Mar. 31, 2011

$n=161186$

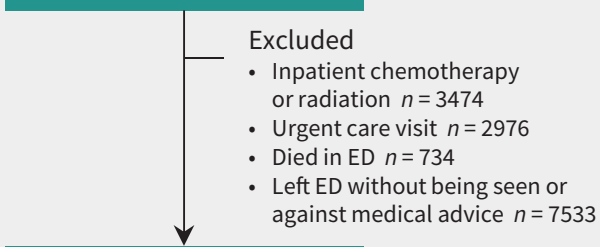

ED visits within $30 \mathrm{~d}$ of

chemotherapy or

radiation treatment

$n=146469$

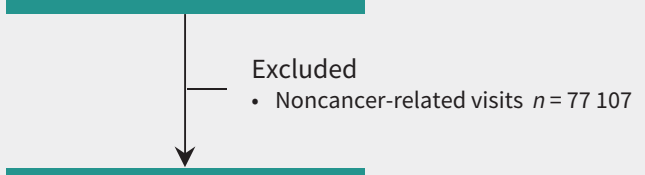

Cancer-related ED visits

within $30 \mathrm{~d}$ of

chemotherapy or

radiation treatment

$n=69362$

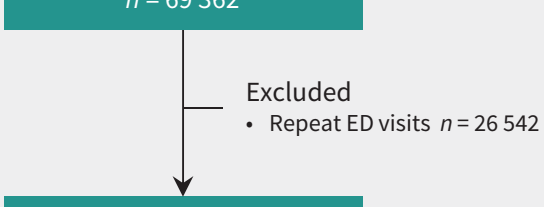

Index ED visits

$n=42820$

Figure 1: Flow chart for participants in the study. Note: ED = emergency department. 
Table 1 (part 1 of 2): Baseline characteristics of patients, by visits to alternative versus original hospital emergency

departments, and by visits to alternative general hospital versus original or cancer centre hospital emergency departments

\begin{tabular}{|c|c|c|c|c|c|c|c|}
\hline Characteristic & $\begin{array}{c}\text { Total no. }(\%)^{\star} \text { of } \\
\text { patients } \\
n=42820\end{array}$ & $\begin{array}{c}\text { No. }(\%)^{\star} \text { of } \\
\text { patients who } \\
\text { visited } \\
\text { alternative } \\
\text { hospital ED } \\
n=16532\end{array}$ & $\begin{array}{c}\text { No. }(\%)^{\star} \text { of } \\
\text { patients who } \\
\text { visited original } \\
\text { hospital ED } \\
n=26288\end{array}$ & $\begin{array}{l}\text { Standardized } \\
\text { difference }\end{array}$ & $\begin{array}{c}\text { No. }(\%)^{\star} \text { of } \\
\text { patients who } \\
\text { visited } \\
\text { alternative } \\
\text { general hospital } \\
\text { ED } \\
n=14691\end{array}$ & $\begin{array}{c}\text { No. }(\%)^{\star} \text { of } \\
\text { patients who } \\
\text { visited original or } \\
\text { cancer centre } \\
\text { hospital ED } \\
n=28129\end{array}$ & $\begin{array}{c}\text { Standardized } \\
\text { difference }\end{array}$ \\
\hline \multicolumn{8}{|l|}{ Demographic } \\
\hline \multicolumn{8}{|l|}{ Age, yr } \\
\hline $21-25$ & $319(0.74)$ & $140(0.9)$ & $179(0.7)$ & 0.02 & $110(0.8)$ & $209(0.7)$ & 0 \\
\hline $26-30$ & $420(1.0)$ & $172(1.0)$ & $248(0.9)$ & 0.01 & $134(0.9)$ & $286(1.0)$ & 0.01 \\
\hline $31-35$ & $694(1.6)$ & $263(1.6)$ & 431 (1.6) & 0 & $226(1.5)$ & $468(1.7)$ & 0.01 \\
\hline $36-40$ & $1227(2.9)$ & $432(2.6)$ & 795 (3.0) & 0.02 & $357(2.4)$ & $870(3.1)$ & 0.04 \\
\hline $41-45$ & $2129(5.0)$ & $782(4.7)$ & $1347(5.1)$ & 0.02 & $654(4.5)$ & $1475(5.2)$ & 0.04 \\
\hline $46-50$ & $3411(8.0)$ & $1232(7.5)$ & $2179(8.3)$ & 0.04 & $1038(7.1)$ & $2373(8.4)$ & 0.05 \\
\hline $51-55$ & $4407(10.3)$ & $1705(10.3)$ & $2702(10.3)$ & 0 & $1481(10.1)$ & $2926(10.4)$ & 0.01 \\
\hline $56-60$ & 5604 (13.1) & 2167 (13.1) & 3437 (13.1) & 0 & $1933(13.2)$ & 3671 (13.1) & 0 \\
\hline $61-65$ & $6233(14.6)$ & 2466 (14.9) & 3767 (14.3) & 0.02 & 2199 (15.0) & $4034(14.3)$ & 002 \\
\hline $66-70$ & $6111(14.3)$ & $2361(14.3)$ & 3750 (14.3) & 0 & 2139 (14.6) & $3972(14.1)$ & 0.01 \\
\hline $71-75$ & 5555 (13.0) & $2161(13.1)$ & 3394 (12.9) & 0.01 & $1982(13.5)$ & 3573 (12.7) & 0.02 \\
\hline $76-80$ & $4001(9.3)$ & $1544(9.3)$ & $2457(9.4)$ & 0 & $1428(9.7)$ & $2573(9.2)$ & 0.02 \\
\hline $81-85$ & $1992(4.7)$ & $800(4.8)$ & $1192(4.5)$ & 0.01 & $737(5.0)$ & $1255(4.5)$ & 0.03 \\
\hline $86-90$ & $615(1.4)$ & $258(1.6)$ & $357(1.4)$ & 0.02 & 229 (1.6) & $386(1.4)$ & 0.02 \\
\hline$\geq 91$ & $102(0.2)$ & $49(0.3)$ & $53(0.2)$ & 0.02 & $44(0.3)$ & $58(0.2)$ & 0.02 \\
\hline \multicolumn{8}{|l|}{ Sex } \\
\hline Female & $22899(53.5)$ & $8291(50.2)$ & $14608(55.6)$ & 0.11 & $7373(50.2)$ & $15526(55.2)$ & 0.10 \\
\hline Male & $19921(46.5)$ & 8241 (49.9) & $11680(44.3)$ & & $7318(49.8)$ & $12603(44.8)$ & \\
\hline \multicolumn{8}{|l|}{ Income quintile } \\
\hline Q1 & 7911 (18.5) & 3049 (18.4) & $4862(18.5)$ & 0 & $2791(19.0)$ & $5120(18.2)$ & 0.02 \\
\hline Q2 & 8797 (20.5) & $3325(20.1)$ & $5472(20.8)$ & 0.02 & $3010(20.5)$ & 5787 (20.6) & 0 \\
\hline Q3 & 8467 (19.8) & 3270 (19.8) & $5197(19.8)$ & 0 & 2918 (19.9) & 5549 (19.7) & 0 \\
\hline Q4 & 8953 (20.9) & 3585 (21.7) & $5368(20.4)$ & 0.03 & $3112(21.2)$ & $5841(20.8)$ & 0.01 \\
\hline Q5 & $8692(20.3)$ & $3303(20.0)$ & $5389(20.5)$ & 0.01 & 2860 (19.5) & $5832(20.7)$ & 0.03 \\
\hline Rural area & $4556(10.6)$ & $2835(17.2)$ & $1721(6.6)$ & 0.35 & 2794 (19.0) & $1762(6.3)$ & 0.42 \\
\hline \multicolumn{8}{|c|}{ Comorbidities and health care utilization } \\
\hline Asthma & $5350(12.5)$ & 2039 (12.3) & 3311 (12.6) & 0.01 & $1811(12.3)$ & 3539 (12.6) & 0.01 \\
\hline COPD & $7682(18.4)$ & 3297 (19.9) & 4565 (17.4) & 0.07 & 3035 (20.7) & $4827(17.2)$ & 0.09 \\
\hline DM & 7911 (18.5) & 3080 (18.6) & $4831(18.4)$ & 0.01 & $2762(18.8)$ & $5149(18.3)$ & 0.01 \\
\hline CAD & $1509(3.5)$ & $658(4.0)$ & $851(3.2)$ & 0.04 & $592(4.0)$ & $917(3.3)$ & 0.04 \\
\hline $\mathrm{CHF}$ & $1951(4.6)$ & $836(5.1)$ & $1115(4.3)$ & 0.04 & $758(5.2)$ & $1193(4.2)$ & 0.04 \\
\hline CVA & $308(0.7)$ & $128(0.8)$ & $180(0.7)$ & 0.01 & $118(0.8)$ & $190(0.7)$ & 0.02 \\
\hline Chronic renal failure & $49(0.1)$ & $23(0.1)$ & $26(0.1)$ & 0.01 & $23(0.2)$ & $26(0.1)$ & 0.02 \\
\hline ADG score, median (IQR) & $8.0(5.0-10.0)$ & $8.0(5.0-10.0)$ & $8.0(5.0-100)$ & 0.01 & $8.0(5.0-10.0)$ & $8.0(5.0-10.0)$ & 0 \\
\hline $\begin{array}{l}\text { Admissions to hospital in } \\
\text { the previous } 2 \mathrm{yr}\end{array}$ & 8928 (20.9) & 3617 (21.9) & $5311(20.2)$ & 0.04 & $3245(22.1)$ & $5683(20.2)$ & 0.05 \\
\hline $\begin{array}{l}\text { ED visits in the previous } \\
2 \mathrm{yr}\end{array}$ & $24960(58.3)$ & $10150(61.4)$ & $14810(56.3)$ & 0.10 & $9131(52.2)$ & $15829(56.3)$ & 0.12 \\
\hline
\end{tabular}


Table 1 (part 2 of 2): Baseline characteristics of patients, by visits to alternative versus original hospital emergency

departments, and by visits to alternative general hospital versus original or cancer centre hospital emergency departments

\begin{tabular}{|c|c|c|c|c|c|c|c|}
\hline Characteristic & $\begin{array}{c}\text { Total no. }(\%)^{\star} \text { of } \\
\text { patients } \\
n=42820\end{array}$ & $\begin{array}{c}\text { No. }(\%)^{\star} \text { of } \\
\text { patients who } \\
\text { visited } \\
\text { alternative } \\
\text { hospital ED } \\
n=16532\end{array}$ & $\begin{array}{c}\text { No. }(\%)^{\star} \text { of } \\
\text { patients who } \\
\text { visited original } \\
\text { hospital ED } \\
n=26288\end{array}$ & $\begin{array}{l}\text { Standardized } \\
\text { difference }\end{array}$ & $\begin{array}{c}\text { No. }(\%)^{\star} \text { of } \\
\text { patients who } \\
\text { visited } \\
\text { alternative } \\
\text { general hospital } \\
\text { ED } \\
n=14691\end{array}$ & $\begin{array}{c}\text { No. }(\%)^{\star} \text { of } \\
\text { patients who } \\
\text { visited original or } \\
\text { cancer centre } \\
\text { hospital ED } \\
n=28129\end{array}$ & $\begin{array}{l}\text { Standardized } \\
\text { difference }\end{array}$ \\
\hline \multicolumn{8}{|l|}{ Cancer } \\
\hline \multicolumn{8}{|l|}{ Type of malignant growth } \\
\hline Solid & $36163(84.5)$ & $14276(86.4)$ & $21887(83.3)$ & \multirow[t]{2}{*}{0.09} & $12750(86.8)$ & $23413(83.2)$ & \multirow[t]{2}{*}{0.10} \\
\hline Hematologic & $6657(15.6)$ & $2256(13.7)$ & $4401(16.7)$ & & $1941(13.2)$ & $4716(16.8)$ & \\
\hline \multicolumn{8}{|l|}{ Treatment type } \\
\hline Radiation & $10834(25.3)$ & $5888(35.6)$ & $4946(18.8)$ & 0.39 & $5325(36.3)$ & 5509 (19.6) & 0.39 \\
\hline Chemotherapy & $28457(66.5)$ & $9395(56.8)$ & $19062(72.5)$ & 0.34 & $8253(56.2)$ & 20204 (71.8) & 0.34 \\
\hline Combination & $3529(8.2)$ & $1249(7.6)$ & $2280(8.7)$ & 0.04 & $1113(7.6)$ & $2416(8.9)$ & 0.04 \\
\hline Palliative care & $10799(25.2)$ & $3881(23.5)$ & $6918(26.3)$ & 0.07 & $3491(23.8)$ & $7308(26.0)$ & 0.05 \\
\hline $\begin{array}{l}\text { No. of wk from diagnosis, } \\
\text { median (IQR) }\end{array}$ & $19.6(11.1-37.3)$ & $19.6(11.1-37.9)$ & $19.4(11.1-36.9)$ & 0.01 & $19.6(11.1-37.6)$ & $19.4(11.1-37.1)$ & 0 \\
\hline \multicolumn{8}{|l|}{ ED visit } \\
\hline Arrived by ambulance & $10425(24.4)$ & $4205(25.4)$ & $6220(23.6)$ & 0.04 & $3718(25.3)$ & $6707(23.8)$ & 0.03 \\
\hline \multicolumn{8}{|l|}{ Triage category } \\
\hline $\begin{array}{l}\text { CTAS 1-2 (highest } \\
\text { acuity) }\end{array}$ & $15757(36.8)$ & $4693(28.4)$ & $11064(42.1)$ & 0.29 & $3793(25.8)$ & $14174(50.4)$ & 0.35 \\
\hline CTAS 3 & $22381(52.3)$ & $9028(54.6)$ & $13353(50.8)$ & 0.08 & 8207 (55.9) & $11964(42.5)$ & 0.11 \\
\hline $\begin{array}{l}\text { CTAS 4-5 (lowest } \\
\text { acuity) }\end{array}$ & $4682(10.9)$ & $2811(17.0)$ & $1871(7.1)$ & 0.32 & $2691(18.3)$ & $1991(7.1)$ & 0.37 \\
\hline \multicolumn{8}{|l|}{ Shift } \\
\hline Day (08:00-15:59) & $19674(46.0)$ & $7823(47.3)$ & $11851(45.1)$ & 0.04 & $7024(47.8)$ & $12650(45.0)$ & 0.06 \\
\hline Evening (16:00-23:59) & $18293(42.7)$ & $6903(41.8)$ & $11390(43.3)$ & 0.03 & $6067(41.3)$ & $12226(43.5)$ & 0.04 \\
\hline Overnight (0:00-07:59) & $4853(11.3)$ & $1806(10.9)$ & 3407 (11.6) & 0.02 & $1600(10.9)$ & $3253(11.6)$ & 0.02 \\
\hline \multicolumn{8}{|l|}{ Day of week } \\
\hline Weekday & $28653(66.9)$ & $10956(66.3)$ & $17697(67.3)$ & 0.02 & $9722(66.2)$ & $18931(67.3)$ & 0.02 \\
\hline Weekend/holiday & $14167(33.1)$ & $5576(33.7)$ & $8591(32.7)$ & & $4969(33.8)$ & $9198(32.7)$ & \\
\hline Cancer centre & $18645(43.5)$ & $1841(11.1)$ & $16804(63.9)$ & 1.25 & - & - & - \\
\hline \multicolumn{8}{|l|}{ Outcomes } \\
\hline Admission to hospital & $19171(44.8)$ & $6518(39.4)$ & $12653(48.1)$ & 0.18 & $5703(38.8)$ & $13468(47.9)$ & 0.18 \\
\hline Mortality, $30 \mathrm{~d}$ & 4659 (10.9) & $1926(11.7)$ & $2733(10.4)$ & 0.04 & $1762(12.0)$ & $2897(10.3)$ & 0.05 \\
\hline Return ED visits & $13912(34.6)$ & $5781(37.2)$ & $8131(33.0)$ & 0.09 & $5196(37.6)$ & $8716(33.0)$ & 0.09 \\
\hline CT imaging & $6487(15.2)$ & $2110(12.8)$ & $4377(16.7)$ & 0.11 & $1760(12.0)$ & $4727(16.8)$ & 0.13 \\
\hline
\end{tabular}

Note: $\mathrm{ADG}=$ Adjusted Diagnostic Group, $\mathrm{CAD}=$ coronary artery disease, $\mathrm{CHF}=$ congestive heart failure, $\mathrm{COPD}=$ chronic obstructive pulmonary disease, $\mathrm{CT}=$ computed tomography, CTAS = Canadian Triage Acuity Scale, CVA = cerebral vascular accident, DM = diabetes mellitus, ED = emergency department, IQR = interquartile range.

*Unless specified otherwise.

In the propensity score-matched cohort, which we used to examine the second independent variable of interest (cancer expertise), 13292 patients (90.5\%) who were seen at an alternative general hospital were matched to patients who visited an original hospital or a cancer centre. Standardized differences were less than 0.10 for all covariates (Table 2), as was matching after emergency department disposition was added to the model for the secondary outcomes (Supplemental Table 3, Appendix 1). Patients seen at alternative general hospitals had significantly lower odds of admission to hospital (OR $0.83,95 \% \mathrm{Cl} 0.79-0.88$ ), 
Table 2 (part 1 of 2): Characteristics of patients seen in the emergency department, matched on visits to alternative versus original hospital emergency departments, and visits to alternative general hospital versus original or cancer centre hospital emergency departments

\begin{tabular}{|c|c|c|c|c|c|c|}
\hline Characteristic & $\begin{array}{c}\text { No. }(\%)^{\star} \text { of } \\
\text { patients who } \\
\text { visited alternative } \\
\text { hospital ED } \\
n=10273\end{array}$ & $\begin{array}{c}\text { No. }(\%)^{\star} \text { of } \\
\text { patients who } \\
\text { visited original } \\
\text { hospital ED } \\
n=10273\end{array}$ & $\begin{array}{l}\text { Standardized } \\
\text { difference of the } \\
\text { mean }\end{array}$ & $\begin{array}{l}\text { No. }(\%)^{\star} \text { of patients } \\
\text { who visited } \\
\text { alternative general } \\
\text { hospital ED } \\
n=13322\end{array}$ & $\begin{array}{l}\text { No. }(\%)^{\star} \text { of patients } \\
\text { who visited original } \\
\text { or cancer centre } \\
\text { hospital ED } \\
n=13322\end{array}$ & $\begin{array}{l}\text { Standardized } \\
\text { difference of } \\
\text { the mean }\end{array}$ \\
\hline \multicolumn{7}{|l|}{ Demographic } \\
\hline \multicolumn{7}{|l|}{ Age, yr } \\
\hline $21-25$ & $68(0.7)$ & $63(0.6)$ & 0 & $100(0.8)$ & $106(0.8)$ & 0 \\
\hline $26-30$ & $105(1.0)$ & $107(1.0)$ & 0 & $128(1.0)$ & $133(1.0)$ & 0 \\
\hline $31-35$ & $167(1.6)$ & $168(1.6)$ & 0 & 208(1.6) & $223(1.7)$ & 0.01 \\
\hline $36-40$ & $295(2.9)$ & $311(3.0)$ & 0.01 & $337(2.5)$ & $343(2.6)$ & 0 \\
\hline $41-45$ & $531(5.2)$ & $546(5.3)$ & 0 & $614(4.6)$ & $621(4.7)$ & 0 \\
\hline $46-50$ & $838(8.2)$ & $854(8.3)$ & 0 & $977(7.3)$ & $968(7.3)$ & 0 \\
\hline $51-55$ & $1104(10.8)$ & $1122(10.9)$ & 0 & $1346(10.1)$ & $1344(10.1)$ & 0 \\
\hline $56-60$ & $1392(13.6)$ & 1364 (13.3) & 0.01 & $1773(13.3)$ & 1764 (13.2) & 0 \\
\hline $61-65$ & $1527(14.9)$ & $1537(15.0)$ & 0 & 1985 (14.9) & $1992(14.9)$ & 0 \\
\hline $66-70$ & $1470(14.3)$ & $1469(14.3)$ & 0 & 1919 (14.4) & 1935 (14.5) & 0 \\
\hline $71-75$ & $1342(13.1)$ & $1335(13.0)$ & 0 & $1767(13.3)$ & 1716 (12.9) & 0.01 \\
\hline $76-80$ & $894(8.7)$ & $844(8.2)$ & 0.01 & $1272(9.6)$ & $1285(9.7)$ & 0 \\
\hline $81-85$ & $409(4.0)$ & $417(4.1)$ & 0 & $656(4.9)$ & $659(5.0)$ & 0 \\
\hline $86-90$ & $117(1.1)$ & $127(1.2)$ & 0.01 & 204 (1.5) & 195 (1.5) & 0 \\
\hline$\geq 91$ & $14(0.1)$ & $9(0.1)$ & 0.01 & $36(0.3)$ & $38(0.3)$ & 0 \\
\hline \multicolumn{7}{|l|}{ Sex } \\
\hline Female & $5701(55.5)$ & $5726(55.7)$ & 0 & $6820(51.2)$ & $6762(50.8)$ & 0.01 \\
\hline Male & $4572(44.5)$ & 4547 (44.3) & & $6502(48.8)$ & $6560(49.2)$ & \\
\hline \multicolumn{7}{|l|}{ Income quintile } \\
\hline Q1 & $1892(18.4)$ & $1870(18.2)$ & 0 & 2479 (18.6) & $2521(18.9)$ & 0.01 \\
\hline Q2 & $2117(20.6)$ & $2079(20.2)$ & 0.01 & $2722(20.4)$ & $2717(20.4)$ & 0 \\
\hline Q3 & $2086(20.3)$ & $2076(20.2)$ & 0 & 2646 (19.9) & $2612(19.6)$ & 0 \\
\hline Q4 & $2175(21.2)$ & $2170(21.1)$ & 0 & $2851(21.4)$ & $2818(21.2)$ & 0 \\
\hline Q5 & $2003(19.5)$ & $2078(20.2)$ & 0.01 & $2624(19.7)$ & 2654 (19.9) & 0 \\
\hline Rural area & $1390(13.5)$ & $1320(12.9)$ & 0.02 & $1771(13.3)$ & $1674(12.6)$ & 0.02 \\
\hline \multicolumn{7}{|c|}{ Comorbidities and health care utilization } \\
\hline Asthma & $1294(12.6)$ & $1297(12.6)$ & 0 & $1652(12.4)$ & $1638(12.3)$ & 0 \\
\hline COPD & $1789(17.4)$ & $1814(17.7)$ & 0 & $2625(19.7)$ & $2587(19.4)$ & 0.01 \\
\hline DM & $1850(18.0)$ & $1903(18.5)$ & 0.01 & $2483(18.6)$ & $2490(18.7)$ & 0 \\
\hline CAD & $349(3.4)$ & $346(3.4)$ & 0 & $514(3.9)$ & $512(3.8)$ & 0 \\
\hline $\mathrm{CHF}$ & $406(4.0)$ & $418(4.1)$ & 0 & $646(4.9)$ & $654(4.9)$ & 0 \\
\hline CVA & $60(0.6)$ & $68(0.7)$ & 0.01 & $105(0.8)$ & $107(0.8)$ & 0 \\
\hline Renal failure & $11(0.1)$ & $9(0.1)$ & 0 & $18(0.1)$ & $18(0.1)$ & 0 \\
\hline ADG score, median (IQR) & $8.0(5.0-10.0)$ & $8.0(5.0-10.0)$ & 0 & $8.0(5.0-10.0)$ & $8.0(5.0-10.0)$ & 0 \\
\hline $\begin{array}{l}\text { Admissions to hospital in the } \\
\text { previous } 2 \mathrm{yr}\end{array}$ & $2180(21.2)$ & $2129(20.7)$ & 0.01 & $2900(21.8)$ & $2886(21.7)$ & 0 \\
\hline Visits to the ED in the previous $2 \mathrm{yr}$ & $6125(59.6)$ & $6059(59.0)$ & 0.01 & 8072 (60.6) & $8140(61.1)$ & 0.01 \\
\hline \multicolumn{7}{|l|}{ Cancer } \\
\hline \multicolumn{7}{|l|}{ Type of malignant growth } \\
\hline Solid & $8493(82.7)$ & 8489 (82.6) & 0 & $11489(86.2)$ & $11489(86.2)$ & 0 \\
\hline Hematologic & $1780(17.3)$ & $1784(17.4)$ & & $1833(13.8)$ & $1833(13.8)$ & \\
\hline \multicolumn{7}{|l|}{ Treatment type } \\
\hline Radiation & $1174(11.4)$ & $1057(10.3)$ & 0.06 & 4388 (32.9) & 4487 (33.7) & 0.01 \\
\hline Chemotherapy & $8216(80.0)$ & 8318 (81.0) & 0.02 & 7877 (59.1) & $7783(58.4)$ & 0.01 \\
\hline Combination & $883(8.6)$ & $898(8.7)$ & 0 & $1057(7.9)$ & $1052(7.9)$ & 0 \\
\hline Palliative care & $2724(26.5)$ & $2557(24.9)$ & 0.03 & 3225 (24.2) & $3271(24.6)$ & 0.01 \\
\hline $\begin{array}{l}\text { No. of wk from diagnosis, } \\
\text { median (IQR) }\end{array}$ & $20.6(11.9-39.9)$ & $19.6(11.3-36.6)$ & 0.01 & $19.6(11.1-38.3)$ & $19.4(10.9-38.7)$ & 0.01 \\
\hline
\end{tabular}


Table 2 (part 2 of 2): Characteristics of patients seen in the emergency department, matched on visits to alternative versus original hospital emergency departments, and visits to alternative general hospital versus original or cancer centre hospital emergency departments

\begin{tabular}{|c|c|c|c|c|c|c|}
\hline Characteristic & $\begin{array}{c}\text { No. }(\%)^{\star} \text { of } \\
\text { patients who } \\
\text { visited alternative } \\
\text { hospital ED } \\
n=10273\end{array}$ & $\begin{array}{l}\text { No. }(\%)^{\star} \text { of } \\
\text { patients who } \\
\text { visited original } \\
\text { hospital ED } \\
n=10273\end{array}$ & $\begin{array}{l}\text { Standardized } \\
\text { difference of the } \\
\text { mean }\end{array}$ & $\begin{array}{l}\text { No. }(\%)^{\star} \text { of patients } \\
\text { who visited } \\
\text { alternative general } \\
\text { hospital ED } \\
n=13322\end{array}$ & $\begin{array}{l}\text { No. }(\%)^{\star} \text { of patients } \\
\text { who visited original } \\
\text { or cancer centre } \\
\text { hospital ED } \\
n=13322\end{array}$ & $\begin{array}{l}\text { Standardized } \\
\text { difference of } \\
\text { the mean }\end{array}$ \\
\hline \multicolumn{7}{|l|}{ ED visit } \\
\hline Arrival by ambulance & $2341(22.8)$ & $2322(22.6)$ & 0 & $3437(25.8)$ & $3461(26.0)$ & 0 \\
\hline \multicolumn{7}{|l|}{ Triage category } \\
\hline CTAS 1-2 (highest acuity) & $3475(33.8)$ & $3615(35.2)$ & 0.02 & $3761(28.2)$ & $3721(27.9)$ & 0.01 \\
\hline CTAS 3 & $5614(54.7)$ & $5532(53.9)$ & 0.01 & $7747(58.2)$ & 7845 (58.9) & 0.02 \\
\hline CTAS 4-5 (lowest acuity) & $1184(11.5)$ & $1126(11.0)$ & 0.01 & $1814(13.6)$ & $1756(13.2)$ & 0.01 \\
\hline \multicolumn{7}{|l|}{ Shift } \\
\hline Day (08:00-15:59) & $4766(46.4)$ & $4748(46.2)$ & 0 & $6251(46.9)$ & $6348(47.7)$ & 0.01 \\
\hline Evening (16:00-23:59) & $4384(42.7)$ & $4413(43.0)$ & 0 & $5570(41.8)$ & $5484(41.2)$ & 0.01 \\
\hline Overnight (0:00-07:59) & $1123(10.9)$ & $1112(10.8)$ & 0 & $1501(11.3)$ & $1490(11.2)$ & 0 \\
\hline \multicolumn{7}{|l|}{ Day of week } \\
\hline Weekday & $6940(67.6)$ & $6883(67.0)$ & 0.01 & $8867(66.6)$ & $8903(66.8)$ & 0 \\
\hline Weekend/holiday & $3333(32.4)$ & $3390(33.0)$ & & $4455(33.4)$ & $4419(33.2)$ & \\
\hline Cancer centre & 1841 (17.9) & 1875 (18.3) & 0.03 & - & - & - \\
\hline
\end{tabular}

Note: $\mathrm{ADG}=$ adjusted diagnostic group, $\mathrm{CAD}=$ coronary artery disease, $\mathrm{CHF}=$ congestive heart failure, $\mathrm{COPD}=$ chronic obstructive pulmonary disease, $\mathrm{CTAS}=\mathrm{Canadian}$ Triage Acuity Scale, $\mathrm{CVA}=$ cerebral vascular accident, $\mathrm{DM}=$ diabetes mellitus, $\mathrm{ED}=$ emergency department, $\mathrm{IQR}=$ interquartile range.

Table 3: Outcomes for the propensity score-matched cohort at alternative versus original hospital emergency departments and alternative general hospital versus original hospital or cancer centre emergency departments

\begin{tabular}{|c|c|c|c|c|c|c|}
\hline Outcome & $\begin{array}{l}\text { No. }(\%) \text { of } \\
\text { patients who } \\
\text { visited } \\
\text { alternative } \\
\text { hospital ED } \\
n=10273\end{array}$ & $\begin{array}{l}\text { No. }(\%) \text { of } \\
\text { patients who } \\
\text { visited original } \\
\text { hospital ED } \\
n=10273\end{array}$ & $\begin{array}{c}\mathrm{OR}^{\star} \\
(95 \% \mathrm{CI})\end{array}$ & $\begin{array}{l}\text { No. }(\%) \text { of patients } \\
\text { who visited } \\
\text { alternative general } \\
\text { hospital ED } \\
n=13322\end{array}$ & $\begin{array}{c}\text { No. }(\%) \text { of } \\
\text { patients who } \\
\text { visited original } \\
\text { or cancer centre } \\
\text { hospital ED } \\
n=13322\end{array}$ & $\begin{array}{c}\mathrm{OR}^{\star} \\
(95 \% \mathrm{CI})\end{array}$ \\
\hline Admission to hospital & 4087 (39.8) & $4710(45.9)$ & $0.78(0.74-0.83)$ & $5344(40.1)$ & 5907 (44.3) & $0.83(0.79-0.88)$ \\
\hline Mortality, $30 \mathrm{~d}$ & $966(9.5)$ & $928(9.1)$ & $1.05(0.95-1.15)$ & $1602(12.1)$ & $1440(10.8)$ & $1.13(1.05-1.22)$ \\
\hline CT imaging & 1347 (13.2) & 1385 (13.6) & $0.97(0.89-1.05)$ & 1673 (12.6) & 2151 (16.2) & $0.74(0.69-0.80)$ \\
\hline
\end{tabular}

significantly higher odds of 30-day mortality (OR $1.13,95 \% \mathrm{Cl}$ 1.05-1.22), and lower odds of CT imaging (OR 0.74, 95\% Cl 0.69$0.80)$. Sensitivity analysis using inverse propensity-treatment weighting did not change results significantly (Supplemental Table 4, Appendix 1).

We determined that 2619 patients (6.1\%) died in hospital, and we excluded these patients from the analysis of return visits to the emergency department. There was a small increase in return visits to the emergency department within 30 days of emergency department or hospital discharge between patients seen at alternative and original hospitals (hazard ratio [HR] 1.06, 95\% Cl 1.03-1.11). Patients seen at alternative general emergency departments also had a small increased hazard of return visits within 30 days ( $\mathrm{HR} 1.07,95 \% \mathrm{Cl} 1.03-1.11$ ) compared with patients seen in emergency departments at original or cancer hospitals.

Sensitivity analyses examining all-cause and repeat visits to emergency departments did not change the results.

\section{Interpretation}

Our results suggest that cancer-related visits to an alternative emergency department (i.e., not associated with the institution where the patient received chemotherapy or radiation therapy) or to an emergency department that was not affiliated with a cancer centre were less likely to result in admission to hospital; 
however, visits to alternative and noncancer centre emergency departments were also associated with a higher risk of shortterm mortality. These findings suggest that the cancer centre affiliation of a hospital rather than continuity of care improves patient outcomes among patients receiving active cancer therapy who require care at the emergency department. We surmise that this could be mediated via increased rates of admission to hospital at these centres.

Patients at both alternative (including general hospitals and cancer centres) and alternative general emergency departments were less likely to be admitted to hospital, and they had a slightly higher hazard of return emergency department visits, compared with patients seen at original hospitals and at original hospitals or cancer centres. In the unmatched cohort, sicker patients (i.e., those with higher acuity triage scores) were more likely to be seen at an original emergency department, as well as at original hospitals or cancer centres. However, in the propensity scorematched cohorts, the groups were well balanced in terms of potential confounders such as presenting illness acuity and ambulance arrival, yet the difference in admissions to hospital between groups persisted.

Multiple factors could explain the decision not to admit patients at alternative and alternative general hospitals. The health care team at an alternative general hospital may have been reluctant to admit a patient undergoing oncology treatment because they did not have oncologists on staff or access to specialized facilities for oncologic care. Patient transfer to another centre is a time-consuming process, which may act as a deterrent. ${ }^{24}$ Even at cancer centres that were not where the patient received their treatment, staff may have wished to avoid admitting a patient who had an established management plan at another hospital, leading to lower admission rates. It is also possible that the lower admission rates could account for the higher hazard of return visits seen at alternative and alternative general hospitals.

Another explanation for the lower admissions at alternative hospitals is that markers of illness acuity were missed at these emergency departments, where the patient and their disease course are not known; without knowing the signs and symptoms that announced worsening of disease in that patient, as well as gaps in knowledge of previous treatments, some patients may have been discharged inappropriately. However, the 30-day mortality was not different between these groups.

Alternative general hospitals were also more likely to discharge patients with cancer than original hospitals or cancer centres, but the 30-day mortality was higher at these sites compared with original hospitals or cancer centres. This suggests that the lack of oncologic expertise at alternative general hospitals may result in missed prognostic factors in patients with cancer, which might have triggered admission to hospital or closer outpatient management had the patient been seen at an original hospital or cancer centre, and, in turn, resulted in the higher mortality seen at alternative general sites. At cancer centres, these prognostic factors may have been more readily recognized owing to the increased experience and expertise with the course of cancer treatment of the health care providers and early preventive care instituted on an outpatient basis. In addition, if an issue was identified, timely follow-up care with the patient's oncology team may have been arranged more easily.

A lack of inpatient care expertise and resources for patients undergoing oncology treatment who are admitted to hospital may have also contributed to the higher 30-day mortality observed among patients seen at alternative general hospitals compared with cancer centres (but not found between original versus alternative sites). There are many satellite cancer centres across Ontario that provide chemotherapy that we considered original hospitals if patients received their treatment at these sites but were not considered a cancer hospital (36\% of patients seen at original hospitals). Although the cancer expertise at satellite centres is not equivalent to that of regional cancer centres, satellite centres must meet several requirements, which includes specialized training in oncology and access to oncologists from cancer centres. ${ }^{19}$ Therefore, satellite centres have more cancer expertise available to them and are likely much more familiar with patients with cancer compared with hospitals that do not provide such services. This element of cancer expertise at these sites may have improved outcomes if patients returned to their original hospital, even if it was not a regional cancer centre.

Previous studies have shown that patients with cancer who are treated at cancer centres have better outcomes. ${ }^{13,15,16}$ Our findings suggest that this may also be true in the emergency setting. Emergency departments not affiliated with a cancer centre may not have the expertise to treat patients with cancer who often have complex medical histories. Emergency department physicians who do not frequently encounter patients with cancer in their practice cannot be expected to know the intricacies of cancer care with minimal to no exposure to the treatment of these patients.

There were no differences observed in CT imaging between patients seen at alternative and original hospitals. However, patients seen at alternative general hospitals were less likely to undergo CT than patients seen at original hospitals or cancer centres. The staff at the cancer centres may have been aware of potential complications that would require an imaging diagnosis. Staff who did not see patients with cancer frequently (at general sites) may have not been aware of these potential complications, leading to more imaging at cancer centres. In addition, cancer centres are typically associated with academic institutions, which may have greater access to imaging modalities overnight and on weekends. The lower rate of imaging at alternative general hospitals could be 1 potential reason for the higher 30-day mortality found among these patients.

\section{Limitations}

Our study has several limitations. The data that we analyzed were from 2006 to 2011 and, as such, represent older data. It is possible that changes in treatment regimens or availability of cancer programs since that time could affect our results. Parts of the approach used to identify cancer-related visits to the emergency department have not been validated; the original 
approach was validated only in patients with breast cancer. If some of the visits to the emergency department were not cancer related, this would likely lead to a conservative bias in our results, because noncancer-related visits would be expected to be managed similarly at all emergency departments.

We were unable to determine which hospitals had access to records from other hospitals; therefore, we were unable to account for this in our analysis. However, the study period was from 2006 to 2011, which is before ConnectingGTA (now ConnectingOntario; www.ehealthontario.on.ca/en/for-healthcare -professionals/connectingontario) became available; therefore, if there was interhospital transfer of information, it was likely to be limited.

We did not account for hospital transfers of patients who were admitted to hospital in the analysis; all outcomes were attributed to the initial emergency department where patients were seen. However, there were a low number of transfers among those who were admitted $(n=521)$, and this was not likely to have affected our results substantially.

We excluded patients who died in the emergency department from the analysis; this could have affected the results for 30-day mortality. However, the number excluded was low, and this was not likely to have altered our results. In addition, removal of these patients would likely result in a conservative bias, because, if these patients were counted as deaths at 30 days, we would expect a greater number of deaths at alternative and alternative general hospitals compared with original hospitals or cancer centres.

Our study examined a heterogeneous group of patients with various types of cancer who were receiving different treatment regimens. Future studies are needed to determine whether our findings are specific to certain cancers or treatment regimens.

Given the study design, we were unable to account for unmeasured confounders, such as subtler measures of illness acuity. Given that sicker patients presented to emergency departments at original hospitals and cancer centres versus those at alternative hospitals and general sites (Table 1), if some residual confounding remained after adjustment, it would mean that the original hospitals and cancer sites saw sicker patients and, therefore, would have worse outcomes. However, the adjusted mortality rate was lower at emergency departments at the cancer centres, making such residual confounding unlikely.

\section{Conclusion}

In this study, we found that almost half of all patients undergoing active treatment for cancer who sought emergency medical care for a cancer-related reason were admitted to hospital. One-third of these patients were seen at an alternative emergency department instead of where the patient's cancer treatment was provided. Patients seen in emergency departments at alternative hospitals that were not associated with a cancer centre were less likely to be admitted to hospital but had higher 30-day mortality. These results suggest that the cancer expertise of an institution is an important predictor of outcomes in the emergency care of patients with cancer.

\section{References}

1. Cancer. Geneva: World Health Organization; 2018. Available: www.who.int/ mediacentre/factsheets/fs297/en/ (accessed 2019 Mar. 29).

2. Leading causes of death, by sex. Ottawa: Statistics Canada; 2014. Available: www. statcan.gc.ca/tables-tableaux/sum-som/l01/cst01/hlth36a-eng.htm (accessed 2015 Sept. 20)

3. Unplanned visits to hospital after adjuvant chemotherapy. Toronto: Cancer Quality Council of Ontario; 2015. Available: https://archive.cancercare.on.ca/ cms/One.aspx?portalld=351209\&pageld=354832 (accessed 2019 Apr. 5).

4. Unplanned hospital visits during radiation therapy. Toronto: Cancer Quality Council of Ontario; 2016. Available: https://archive.cancercare.on.ca/cms/One. aspx?portalld=351209\&pageld=354838 (accessed 2019 Apr. 5).

5. Enright K, Grunfeld E, Yun L, et al. Population-based assessment of emergency room visits and hospitalizations among women receiving adjuvant chemotherapy for early breast cancer. J Oncol Pract 2015;11:126-32.

6. Staples JA, Thiruchelvam D, Redelmeier DA. Site of hospital readmission and mortality: a population-based retrospective cohort study. CMAJ Open 2014;2:E77-85.

7. Brooke BS, Goodney PP, Kraiss LW, et al. Readmission destination and risk of mortality after major surgery: an observational cohort study. Lancet 2015;386:884-95.

8. Tsai TC, Orav EJ, Jha AK. Care fragmentation in the postdischarge period: Surgical readmissions, distance of travel, and postoperative mortality. JAMA Surg 2015;150:59-64.

9. Wasson JH, Sauvigne AE, Mogielnicki RP, et al. Continuity of outpatient medical care in elderly men. A randomized trial. JAMA 1984;252:2413-7.

10. Menec VH, Sirski M, Attawar D. Does continuity of care matter in a universally insured population? Health Serv Res 2005;40:389-400.

11. Barbas AS, Turley RS, Mantyh CR, et al. Effect of surgeon specialization on long-term survival following colon cancer resection at an $\mathrm{NCl}$-designated cancer center. J Surg Oncol 2012;106:219-23.

12. Simunovic M, Rempel E, Theriault ME, et al. Influence of hospital characteristics on operative death and survival of patients after major cancer surgery in Ontario. Can J Surg 2006;49:251-8.

13. Birkmeyer NJ, Goodney PP, Stukel TA, et al. Do cancer centers designated by the national cancer institute have better surgical outcomes? Cancer 2005;103:435-41.

14. Onega T, Duell EJ, Shi X, et al. Influence of $\mathrm{NCl}$ cancer center attendance on mortality in lung, breast, colorectal, and prostate cancer patients. Med Care Res Rev 2009;66:542-60.

15. Paulson EC, Mitra N, Sonnad S, et al. National cancer institute designation predicts improved outcomes in colorectal cancer surgery. Ann Surg 2008;248: 675-86.

16. Wolfson JA, Sun CL, Wyatt LP, et al. Impact of care at comprehensive cancer centers on outcome: results from a population-based study. Cancer 2015;121:3885-93.

17. Krzyzanowska MK, Enright K, Moineddin R, et al. Can chemotherapy-related acute care visits be accurately identified in administrative data? J Oncol Pract 2018;14:e51-8.

18. Common toxicity criteria manual. Bethesda (MD): National Cancer Institute; 1999.

19. Regional systemic treatment plan. Toronto: Cancer Care Ontario; 2009.

20. Johns Hopkins ACG case-mix adjustment system. Baltimore (MD): John Hopkins University; 2012. Available: https://www.hopkinsacg.org/ (accessed 2019 Mar. 29). 
21. Beveridge $\mathrm{R}$, Clarke $\mathrm{B}$, Janes $\mathrm{L}$, et al. Implementation guidelines for the Canadian emergency department Triage \& Acuity Scale (CTAS); 1998. Available: http://ctas-phctas.ca/wp-content/uploads/2018/05/ctased16_98.pdf (accessed 2019 Mar. 29).

22. Austin PC. A comparison of 12 algorithms for matching on the propensity score. Stat Med 2014;33:1057-69.
23. Austin PC. Balance diagnostics for comparing the distribution of baseline covariates between treatment groups in propensity-score matched samples. Stat Med 2009;28:3083-107.

24. Hains IM, Marks A, Georgiou A, et al. Non-emergency patient transport: What are the quality and safety issues? A systematic review. Int J Qual Health Care 2011;23:68-75.
Competing interests: Monika Krzyzanowska has received an unrestricted education grant from Ipsen, an honourarium for attending an advisory board meeting at Eisai, and other support as a site principal investigator for clinical trials for Eisai and Excelixis. No other competing interests were declared.

This article has been peer reviewed.

Affiliations: Schwartz/Reisman Emergency Medicine Institute (Grewal), Sinai Health System; ICES (Grewal, Sutradhar, Krzyzanowska, Redelmeier, Atzema); University Health Network (Krzyzanowska); Sunnybrook Health Sciences Centre (Redelmeier, Atzema); Division of Emergency Medicine (Grewal, Atzema), Divisions of Medical Oncology and Hematology (Krzyzanowska), and Division of General Internal Medicine (Redelmeier), Department of Medicine, University of Toronto; Institute for Health Policy, Management \& Evaluation (Sutradhar, Redelmeier, Atzema), University of Toronto, Toronto, Ont.

Contributors: All of the authors contributed to study conception and design, and the acquisition, analysis or interpretation of data. Keerat Grewal and Clare Atzema drafted the manuscript. Keerat Grewal had full access to all the data in the study and performed the statistical analysis. Clare Atzema supervised the study. All of the authors contributed to editing and revised the manuscript critically for important intellectual content, gave final approval of the version to be published and agreed to be accountable for all aspects of the work.

Data sharing: The data set from this study is held securely in coded form at ICES. Although data-sharing agreements prohibit ICES from making the data set publicly available, access may be granted to those who meet prespecified criteria for confidential access (available at www.ices.on.ca/DAS)

Disclaimer: This study made use of de-identified data from the ICES Data Repository, which is managed by the ICES with support from its funders and partners: Canada's Strategy for Patient-Oriented Research (SPOR), the Ontario SPOR Support Unit, the Canadian Institutes of Health Research and the Government of Ontario. This study was supported through pro- vision of data by the ICES and Cancer Care Ontario (CCO) and through funding support to ICES from an annual grant by the Ministry of Health and Long-Term Care and the Ontario Institute for Cancer Research. The opinions, results and conclusions reported in this paper are those of the authors. No endorsement by ICES or any of its funders or partners is intended or should be inferred. Parts of this material are based on data and information compiled and provided by Canadian Institute for Health Information (CIHI). However, the analyses, conclusions, opinions and statements expressed herein are those of the author, and not necessarily those of $\mathrm{ClHI}$. Parts of this material are based on data and information provided by CCO. The opinions, results, view, and conclusions reported in this paper are those of the authors and do not necessarily reflect those of $\mathrm{CCO}$. No endorsement by $\mathrm{CCO}$ is intended or should be inferred.

Accepted: Mar. 31, 2019

Correspondence to: Keerat Grewal, keerat. grewal@sinaihealthsystem.ca 